\title{
Individualized Sellar Floor Reconstruction Method and Nursing Strategy for the Intraoperative Cerebrospinal Fluid Leakage Caused by Neuroendoscopic Transsphenoidal Pituitary Adenoma Resection
}

\author{
Xiaoqun Chen'1, Siting Fan², Shuo Yang1, Jiayu Gu³, Qinqin Zhao1, Zhihuan Zhou ${ }^{{ }^{*}}$ \\ ${ }^{1}$ Department of Neurosurgery, Sun Yat-sen University Cancer Center, State Key Laboratory of Oncology in South China, \\ Collaborative Innovation Center for Cancer Medicine, Guangzhou, China \\ ${ }^{2}$ Department of Gastric Surgery, Sun Yat-sen University Cancer Center, State Key Laboratory of Oncology in South China, \\ Collaborative Innovation Center for Cancer Medicine, Guangzhou, China \\ ${ }^{3}$ Department of Head and Neck Surgery, Sun Yat-sen University Cancer Center, State Key Laboratory of Oncology \\ in South China, Collaborative Innovation Center for Cancer Medicine, Guangzhou, China \\ Email: *zhouzhh@sysucc.org.cn
}

How to cite this paper: Chen, X.Q., Fan, S.T., Yang, S., Gu, J.Y., Zhao, Q.Q. and Zhou, Z.H. (2021) Individualized Sellar Floor Reconstruction Method and Nursing Strategy for the Intraoperative Cerebrospinal Fluid Leakage Caused by Neuroendoscopic Transsphenoidal Pituitary Adenoma Resection. Open Journal of Nursing, 11, 367-377.

https://doi.org/10.4236/ojn.2021.115032

Received: April 14, 2021

Accepted: May 23, 2021

Published: May 26, 2021

Copyright $\odot 2021$ by author(s) and Scientific Research Publishing Inc. This work is licensed under the Creative Commons Attribution International License (CC BY 4.0).

http://creativecommons.org/licenses/by/4.0/ (c) (i) Open Access

\begin{abstract}
Objective: To explore the clinical effect of and the method and nursing strategy for sellar reconstruction for intraoperative cerebrospinal fluid (CSF) leakage in pituitary adenoma resection under neuroendoscopy. Methods: The data from 20 cases of intraoperative CSF leak caused by transsphenoidal pituitary adenoma resection were retrospectively analyzed. Six patients were treated with mucosal flap and artificial dural reconstruction of sellar (simple sellar floor reconstruction). In 45 patients, autologous fat, fascia lata, and artificial dura were used to repair and reconstruct the sellar floor (multilayer sellar floor reconstruction). After the operation, all patients underwent follow-up for 6 - 24 months. Results: Fifty patients were followed up without CSF leakage. One patient was cured after leakage of CSF through the lumbar cistern 1 month after discharge. Conclusion: For patients with CSF leakage during neuroendoscopic transsphenoidal pituitary adenoma resection, individualized sellar floor reconstruction should be adopted according to the degree of CSF leakage and the size of the sellar floor defect. Strict nursing measures can effectively prevent CSF leakage and reduce postoperative complications.
\end{abstract}

\section{Keywords}

Pituitary Adenoma, Neuroendoscopic, Intraoperative Cerebrospinal Fluid 
Leakage, Sellar Floor Reconstruction, Nursing Strategy

\section{Introduction}

The pituitary adenoma is a common benign intracranial tumor, and the neuroendoscopic transsphenoidal approach is the preferred surgical treatment for pituitary adenoma [1]. Cerebrospinal fluid (CSF) leakage is the most common complication of pituitary adenoma, and the incidence of intraoperative CSF leakage is gradually increasing. Timely intraoperative sellar floor reconstruction is the key link to prevent CSF leakage, and timely sellar floor reconstruction can effectively reduce postoperative CSF leakage [2]. In this research, we retrospectively analyzed the clinical data of 51 patients with CSF leakage during neuroendoscopic pituitary adenoma resection between November 2018 and January 2021. All of these patients underwent sellar floor reconstruction in time during the operation, and implement rigorous nursing strategy after surgery, and achieved good clinical effect. Now the reconstruction methods and nursing strategy of sellar floor reconstruction are retrospectively reported as follows.

\section{Clinical Data and Methods}

\subsection{Clinical Data}

\subsubsection{Baseline Demographic and Disease Information}

The clinical data from 51 patients with CSF leakage undergoing neuroendoscopic repair from November 2018 to January 2021 were selected from patients with pituitary adenoma treated by neuroendoscopic sinus surgery in our department. Among them, 26 patients were male and 25 patients were female; with an age range of $30-76$ and an average age of $50.71 \pm 10.532$ years. All patients underwent preoperative neuroendocrine examination and pituitary MRI examination to determine tumor size, invasion site, and sellar diaphragm conditions.

\subsubsection{Imaging Results}

There were 46 cases of large adenoma (10 - $40 \mathrm{~mm}$ in diameter) and 5 cases of giant adenoma ( $>40 \mathrm{~mm}$ in diameter). Among them, there were $5.88 \%$ ( 3 cases) in Knops Grade 0, 27.45\% (14 cases) in Grade I, 37.25\% (19 cases) in Grade II, 19.61\% (10 cases) in Grade III, and 9.81\% (5 cases) in Grade IV; Knops $\geq$ Grade III was invasive pituitary adenoma.

\subsubsection{Clinical Manifestations}

There were 9 cases of headache, 27 cases of visual dysfunction, 1 case of lactation, 4 cases of acromegaly, and 1 case of menstrual disorder. 8 patients had hypertension, 1 patient had diabetes mellitus, 1 patient had heart disease, and 1 patient had hyperthyroidism. 4 patients had recurrence after surgical treatment, and 2 patients had a pituitary tumor with stroke.

\subsubsection{Pathological Types}


There were 33 patients with nonfunctional adenoma (NFPA), growth hormone (GH) adenoma (7 cases), prolactin (PRL) adenoma (4 cases), thyroid-stimulating hormone (TSH) adenoma (1 case), gonadotropin (FSH) adenoma (2 cases), adrenergic hormone (ACTH) adenoma (2 cases), GH and ACTH adenoma mixed (1 case), TSH and FSH adenoma mixed (1 case).

\subsection{Methods}

\subsubsection{Preoperative Preparation}

All patients underwent neuroendoscopic transsphenoidal pituitary adenoma resection under general anesthesia. Eye drops of $0.25 \%$ chloramphenicol were used to drip the bilateral nasal cavity 3 days before the operation, nasal hair was cut off 1 day before the operation, and the nasal cavity was cleaned with normal saline.

\subsubsection{The Procedure}

The patient was placed in the supine position, with the head supported to fix the head 15 degrees to the left and slightly backward. The operative area was disinfected, and the table was laid. The right nasal approach was selected. First, the internal conditions of the nasal cavity were explored with a nasal endoscope, and the bilateral turbinate mucosa was contracted using $0.1 \%$ adrenaline. Antecedent right nasal septum pedicled mucosal flap was generated. The sphenoid sinus opening was explored $1.5 \mathrm{~cm}$ above the left posterior nostril, and the mucosal flap at the posterior part of the left nasal septum was made electrically, turning downward. The exfoliator was fractured at the base of the nasal septum, and the anterior wall of the bilateral sphenoid sinus was separated by pushing. The bone of the nasal septum root and the anterior wall of the sphenoid sinus was removed, and the opening of the sphenoid sinus bone window was approximately $2 \times 2 \mathrm{~cm}$. The mucosa of the sphenoid sinus was removed to expose the base of the saddle. After grinding to the bottom of the saddle bone, the dura mater was exposed, hydrogen peroxide disinfection was used to wash the area. The dura mater was revealed with a bright electric light, and a " $U$ " incision exposed the tumor after the dura mater. Endoscopy was used to distinguish normal tissue and pituitary tumor, and tumor forceps were used to take part of the first tumor tissue for pathology examination. To reoccupy the curet of pituitary adenoma, the attractor will block all tumor resection and detect a tumor cavity. If there was a residual tumor, a completely resected tumor sellar was observed, and the sellar was complete. After the tumor was completely removed, the lumen was filled with a gelatine sponge, and the base of the sellar was sealed with biological protein glue and artificial dura. Note that the suprasellar part of the tumor should be removed gently to avoid damage to the arachnoid at the stalk of the sellar septum funnel. Once the arachnoid of the sellar septum hole is damaged, CSF leakage will occur. If the clear fluid was found to flow out during the operation, CSF leakage repair occurred, and reconstruction of the base of the sellar floor was performed. The mucosa of the anterior wall of the sphenoid sinus was res- 
tored, and the hematocele of the nasal cavity was removed by the aspirator. The water sac catheter (silica gel urethral catheter FR16) was placed into the sphenoid sinus of the nasal cavity and placed above the mucosal flap. Six milliliters of normal saline was injected into the catheter to support the base of the sellar, compress the nasal cavity, and stop the bleeding, and the turbinate was restored. Antibiotic anti-infection treatment was given postoperatively, and the water sac catheter was removed one week postoperatively.

\subsubsection{Grading of Intraoperative CSF Leakage}

During the operation, clear fluid was observed to flow from inside and/or beside the saddle. A $5 \mathrm{~mm}$ aspirator head could be used for measurement at the arachnoid opening. By judging the size of the arachnoid opening, it was possible to judge the grade of CSF leakage: 0 is no intraoperative CSF leakage, Grade 1 is low-flow CSF leakage (arachnoid opening $<5 \mathrm{~mm}$ ), and Grade 2 is high-flow CSF leakage (arachnoid opening $\geq 5 \mathrm{~mm}$ ).

\subsubsection{Sellar Floor Reconstruction}

According to the degree of CSF leakage and saddle defect, individualized sellar floor repair was performed immediately after tumor resection. The exact location of the CSF leakage was determined by neuroendoscopy. In the case of low-flow CSF leakage, no obvious defect of the sellar septum, or small leakage, simple sellar floor reconstruction was feasible. A gelatine sponge was used to fill the tumor cavity, a mucosal flap and artificial dura mater were used to repair the leakage, and biological protein was used to cement the leakage. For patients with high-flow CSF leakage or obvious defects of the sellar septum with large leakage, multilayer saddle base combined reconstruction was performed.

The specific method was to crush autologous lateral thigh or abdominal muscle into sarcoplasm to fill the tumor cavity to form a hydrophobic layer. According to the size and shape of the defect of the sellar base, the fascia lata was cut, the cut fascia lata was covered on the dural damage of the seller base, biological protein glue was used to seal and reinforce the defect, and artificial meninges were attached. Finally, a pedicled nasal septum mucosal flap was placed over the base of the sellar, and the base of the sellar was supported by a water sac catheter. For 3 cases of high-flow CSF leakage during the operation and transient CSF leakage after the operation, based on reinforcing the leakage repair, a lumbar cistern drainage tube was used to continuously drain the CSF to reduce the stimulation of the CSF to the reconstruction layer of the saddle base. At the same time, the CSF leakage healed and was discharged after $1-2$ weeks of absolute bed rest.

\section{Postoperative Care}

\subsection{Observation of the Condition}

Changes in the patient's consciousness and vital signs should be closely observed after the operation. If the patient exhibits consciousness disorder or agitation or 
an unequally large pupil, the possibility of cerebral hernia should be considered, and the consciousness should be immediately informed to cooperate with the rescue. For patients with indwelling lumbar cistern drainage tubes, attention should be paid to whether there is drainage tube blockage, low cranial pressure, intracranial infection, intracranial hematoma, and other complications. The color, character, and quantity of drainage solution in each shift should be recorded. Attention should be paid to the patient's chief complaint; if the patient has a headache, nausea, vomiting, and other manifestations, the doctor should be informed in time and craniocerebral CT examination performed if necessary. Urine volume, electrolyte changes, and hormone levels were recorded to observe whether there was CSF leakage. If CSF leakage occurs, absolute bed rest, lumbar cistern drainage, surgical repair, and other methods should be adopted to promote the healing of the leakage.

\subsection{Care of CSF Leakage}

The occurrence of CSF leakage was observed closely after the operation, and the changes in color, character, and quantity of nasal effusion were observed and recorded. If there was clear liquid with a "salty smell" from the nasal cavity, pharyngeal wall outflow alerted to the possibility of postoperative CSF leakage. Clinically, CSF leakage can also be qualitatively judged by urine glucose dipstick, and if positive, it can be judged as CSF leakage [3]. For patients with CSF leakage, bed rest with a high head position should be taken, and the occurrence of low cranial pressure symptoms, such as headache, nausea, vomiting, etc. should be noted. Once intracranial hypotension syndrome occurs, the lumbar cistern drainage tube should be promptly clamped, and the doctor should be immediately informed. If high fever and headache occur, the intracranial infection may occur.

\subsection{Nasal Care}

In the past, iodoform yarn was used for compression to stop bleeding, but this method easily caused uneven pressure in the compression part; at the same time, it was easy to tear off the scabbed skin when pulling out the yarn, which may cause defects of the saddle base that had been repaired successfully again and cause CSF leakage [4]. The water sac catheter supports the sellar base and can reduce the risk of CSF leakage [5]. The water sac catheter on the operative side of the nasal cavity of the patient should be properly fixed after surgery. Patients should be informed to avoid actions that increase nasal pressure within 1 to 2 months after surgery, such as forcible nose blowing or coughing, nose picking, excessive bending, bending, or holding their breath. Nasal irrigation drops and other operations are prohibited. Pulling the water sac catheter should be avoided. If the catheter falls off, it should not be returned to the nasal cavity to avoid infection.

Generally, the water sac catheter should be removed within 7 days after surgery according to the patient's recovery. After the catheter was removed, the pa- 
tient's nasal conditions were observed, and the patient was encouraged to get out of bed as soon as possible. For 3 months after discharge, the patient should avoid coughing, sneezing, blowing nose, and nose picking.

\subsection{Postural Care}

Patients with CSF leakage should stay in bed for 1 - 2 weeks in a supine position or lateral position, raise the head of the bed 15 - 30 degrees, promote intracranial venous reflux, reduce brain edema, relieve postoperative headache, and use gravity to move intracranial tissue to the skull base to promote leakage healing [6]. Because the patient stays in bed for a long time, for those with clear consciousness and normal limb muscle strength, the patient should be guided to exercise on the bed, which can promote venous reflux of the lower extremity and prevent deep venous thrombosis of the lower extremity. Ankle pump exercise can promote blood circulation of the lower limbs and effectively prevent the occurrence of deep venous thrombosis. For patients with long-term bed rest, surgery, and tumors, nurses should actively guide effective ankle pump exercise [7].

\subsection{Nursing of the Lumbar Cistern Drainage Tube}

The lumbar cistern drainage tube is an important nonsurgical treatment for CSF leakage. It has the advantages of minimal trauma, simple operation, promotion of leakage healing, shortening the recovery time, and a low incidence of complications such as retrograde infection [8] [9]. To ensure that the height of the drainage tube on the external auditory canal was $10-15 \mathrm{~cm}$, folding was avoided, the drainage tube was pulled, the drainage tube was determined to be unobstructed, and the drainage tube height was adjusted in time to avoid the occurrence of brain hernia induced by low intracranial pressure syndrome caused by excessive drainage. The drainage speed should be controlled at $10-15 \mathrm{ml} / \mathrm{h}$, and the drainage flow should be $150-350 \mathrm{ml} / \mathrm{d}$ to avoid causing low intracranial pressure. The retention time is generally within 7 days [10] [11]. The color of the drainage fluid is usually colorless and clear. If it becomes bright red or flocculent, intracranial hemorrhage or intracranial infection should be considered. When dumping drainage fluid, the principle of aseptic operation should be strictly implemented to avoid retrograde infection.

\section{Results}

\subsection{Postoperative Results (Table 1)}

Surgical results: soft quality 29 cases, tough quality 22 cases; low-flow CSF leakage 46 cases, high-flow CSF leakage 5 cases; total tumor resection 49 cases, secondary total resection 2 cases.

Postoperative endocrine status: 5 cases GH adenoma, postoperative GH decreased to the normal level; 3 cases PRL adenoma, postoperative PRL decreased to the normal level; 2 cases of ACTH adenoma, postoperative cortisol decreased. 
Postoperative imaging: a postoperative re-examination of craniocerebral MRI, including 49 cases of total tumor resection without residue, 2 cases of secondary total resection, 1 case with a small amount of residual sellar, and 1 case with a small amount of residue on the sellar.

Table 1. Clinical data for the 51 patients.

\begin{tabular}{|c|c|c|}
\hline \multicolumn{2}{|c|}{ Clinical data } & \multirow{2}{*}{$\begin{array}{c}\text { The number of cases (\%) } \\
26(50.98)\end{array}$} \\
\hline S & male & \\
\hline Jex & female & $25(49.02)$ \\
\hline \multirow{3}{*}{ Age } & $<40$ years & $9(17.64)$ \\
\hline & $40-59$ years & $34(66.67)$ \\
\hline & $\geq 60$ years & $8(15.69)$ \\
\hline \multirow{5}{*}{ Preoperative complications } & hypertension & $8(15.69)$ \\
\hline & diabetes & $1(1.96)$ \\
\hline & thyroid disease & $1(1.96)$ \\
\hline & heart disease & $1(1.96)$ \\
\hline & none & $41(80.39)$ \\
\hline \multirow{2}{*}{ Incidence } & starting & $46(90.2)$ \\
\hline & relapse & $5(9.80)$ \\
\hline \multirow{2}{*}{ Tumor size } & large adenoma & $46(90.20)$ \\
\hline & giant adenoma & $5(9.80)$ \\
\hline \multirow{2}{*}{ Tumour texture } & soft & $29(56.86)$ \\
\hline & tough & $22(43.14)$ \\
\hline \multirow{2}{*}{ Tumour blood supply } & rich blood supply & $28(54.90)$ \\
\hline & blood for general & $23(45.10)$ \\
\hline \multirow{5}{*}{ Knops classification } & 0 & $3(5.88)$ \\
\hline & I & $14(27.45)$ \\
\hline & II & $19(37.25)$ \\
\hline & III & $10(19.61)$ \\
\hline & IV & $5(9.81)$ \\
\hline \multirow{2}{*}{ The extent of tumor resection } & full cut & 49 (96.08) \\
\hline & subtotal cut & $2(3.92)$ \\
\hline \multirow{8}{*}{ Pathological classification } & ACTH & $2(3.92)$ \\
\hline & FSH & $2(3.92)$ \\
\hline & GH & $7(13.73)$ \\
\hline & GH and ACTH mixed & $1(1.96)$ \\
\hline & NFPA & $33(64.71)$ \\
\hline & PRL & $4(7.84)$ \\
\hline & TSH & $1(1.96)$ \\
\hline & TSH and FSH mixed & $1(1.96)$ \\
\hline
\end{tabular}




\begin{tabular}{ccc}
\hline $\begin{array}{c}\text { Degree of cerebrospinal fluid } \\
\text { leakage }\end{array}$ & $\begin{array}{c}\text { low-flow leakage } \\
\text { high-flow leakage }\end{array}$ & $46(90.20)$ \\
$5(9.80)$
\end{tabular}

Postoperative complications: 10 patients with CSF leakage were repaired by sellar floor reconstruction and controlled by lumbar cistern drainage. CSF leakage with intracranial infection occurred in 1 case and was cured and discharged by an indwelling lumbar cistern drainage tube.

\subsection{Postoperative Follow-up}

Fifty-one patients were followed up for 6 to 24 months after surgery, and the follow-up methods were outpatient and telephone follow-up. One case of nonfunctional pituitary adenoma was readmitted to the hospital one week after discharge because of CSF rhinorrhoea and intracranial infection after sneezing. One case of nonfunctional pituitary adenoma was readmitted half a month after discharge because of nasal bleeding after sneezing. After returning to the hospital, nasal cavity exploration and hemostasis were cured, and the patients were discharged. After follow-up, no CSF leakage or epistaxis occurred again.

\section{Discussion}

CSF leakage is the most common complication of transsphenoidal pituitary adenoma surgery [12], and its occurrence is related to many factors, such as tumor location, size, tumor invasiveness, and tumor blood supply [13] [14]. Although most have low-flow CSF leakage, the risk of retrograde intracranial infection secondary to CSF leakage is high, which not only exacerbates the patient's condition and even endangers life but also prolongs hospital stay and increases medical costs. Therefore, it is important to reconstruct the skull base properly and effectively to avoid CSF leakage and intracranial infection.

\subsection{CSF Leakage}

The cause of CSF leakage during surgery is related to the degree of damage to the sellar septum, the size of the tumor, the skill of the operator, the texture of the tumor, secondary operation, tumor stroke, or cystic degeneration [15] [16]. In this study, 4 cases were giant adenomas, which were invasive to the suprasellar region, resulting in thin and easily damaged parts of the septum. In these 4 patients, intraoperative sellar reconstruction was adopted, and the postoperative effect was good, and there was no CSF leakage.

To reduce the occurrence of postoperative cerebrospinal fluid leakage, the most important thing is to adopt different intraoperative methods of reconstruction of the sellar area according to the situation of the sellar defect and the degree of CSF leakage. With the innovation of skull base reconstruction technology, the research and development of repair materials, and the continuous accu- 
mulation of the surgeon's experience. These all further reduce the occurrence of CSF leakage after surgery.

\subsection{Intraoperative Sellar Floor Reconstruction}

The method of intraoperative sellar floor reconstruction has a decisive effect on the occurrence of postoperative CSF leakage. Generally, according to the degree of CSF leakage, there are three methods in clinical practice. The first method is a gelatine sponge. This method is suitable for complete protection of the sellar septum and small defects of the sellar floor. The second is mucosal flap reconstruction, which is usually repaired with a pedicle nasal septum mucilage flap. The repair material has the advantages of complete blood supply, fast growth, wide-coverage, tight fit, and so on [17]. It is usually suitable for low-flow CSF leakage or small sellar defects. The third method is multilayer reconstruction, which is often used to repair the sellar floor with autologous fat, fascia lata, and pedicled mucosal flap.

In this study, for low-flow CSF leakage or no obvious sellar damage, mucosal flap sellar floor reconstruction was adopted, while for severe cases of high-flow CSF leakage or sellar septum damage, multilayer sellar floor combined mucosal flap reconstruction was used. Autologous fat, fascia lata, mucosal flap, and artificial dura can form a tight hydrophobic barrier. The repair success rate is high. It is an effective sellar floor reconstruction method commonly used in clinics at present [18]. In 4 cases, the failure of high-flow CSF leak repair may be due to a large amount of bone destruction during the operation in some patients, which cannot provide strong sellar support during sellar repair, as the results of Ma Dongbin et al. show [19].

Due to the high success rate of multilayer sellar floor reconstruction, domestic and foreign literature has reported that the use of self-fat, fascia lata, and bio glue closed multilayer sellar floor reconstruction has a high repair success rate of up to $94 \%$ - 98.4\%, effectively reducing CSF leakage [20] [21].

\subsection{Nursing Care of CSF Leakage after the Operation}

Strict postoperative nursing is an important factor affecting CSF leakage repair. Nurses should guide patients to avoid increasing intracranial pressure and avoid emotional agitation. Usually, bed rest and lumbar cistern drainage tube support treatment are performed to actively prevent intracranial infection.

\section{Summary}

In summary, for patients with CSF leakage in pituitary adenoma, an individualized sellar floor reconstruction strategy should be adopted according to the degree of sellar septum defect and CSF leakage classification, combined with strict nursing after the operation, which can effectively improve the effect of CSF leakage repair. 


\section{Conflicts of Interest}

The authors declare no conflicts of interest regarding the publication of this paper.

\section{References}

[1] Zhang, Y., Wang, Z., Liu, Y., et al. (2007) Treatment of Pituitary Tumors via Endoscopic Endonasal Transsphenoidal Approach. Chinese Journal of Micro Invasive Neurosurgery, 12, 51-54.

[2] Gao, F., Heng, L., Jia, D., et al. (2014) A Novel Sellar Floor Reconstruction Method in Neurons Cope-Assisted Transsphenoidal Microsurgery for Pituitary Adenomas. Chinese Journal of Clinical Neurosurgery, 19, 403-405.

[3] Lu, C. (2011) Experience in Nursing of Single Nostril Transsphenoidal Pituitary Adenoma. Chinese Journal of General Practice, 9, 1485-1486.

[4] Xu, X., Shi, W., Ni, L. and Chen, J. (2016) Investigation of Reasons and Treatment of Cerebrospinal Fluid Leakage after Reconstruction of Basilar Pituitary Fossa in Pituitary Adenoma Surgery by Neural Endoscopy. Journal of Clinical Neurosurgery, 13, 86-92.

[5] Kulwin, C., Schwartz, T.H. and Cohengadol, A.A. (2013) Endoscopic Extended Transsphenoidal Resection of Tuberculum Sellar Meningiomas: Nuances of the Neurosurgical Technique. Neurosurgical Focus, 35, E6. https://doi.org/10.3171/2013.8.FOCUS13338

[6] Shen, J. and Li, S. (2020) Perioperative Nursing Study of Cerebrospinal Fluid Rhinorrhea Repair under Nasal Endoscopy. Today Nurse, 27, 84-87

[7] Lu, X., Wang, J., Sun, J., et al. (2021) Advances in Prevention of Deep Venous Thrombosis by Ankle Pump Exercise. Chinese Journal of Modern Nursing, 27, 447-450

[8] Ren, H., Song, L., Wu, C., et al. (2005) Treatment of Traumatic Cerebrospinal Fluid Leakage with Continuous External Drainage of Lumbar Cistern. Chinese Journal of Clinical Neurosurgery, 10, 318-319.

[9] Li, W. and Zhang, T. (2021) Observation of the Effect of Lumbar Cistern Drainage on Refractory Cerebrospinal Fluid Leakage. Journal of Diseases Monitor \& Control, 15, 37-39.

[10] Zhang, J., Zhu, Z., Sun, S., et al. (2018) Application of Postoperative External Drainage of the Lumbar Cistern for Patients with Brain Tumor. Guangdong Medical Journal, 39, 2131-2133.

[11] Jia, F., Ruan, S. and Fu, L. (2013) Clinical Efficacy of Intrathecal Injection of Vancomycin in the Treatment of Postoperative Intracranial Infections in Neurosurgery Department Patients. Chinese Journal of Nosocomiology, 23, 5525-5526, 5529.

[12] Lam, G., Mehta, V. and Zada, G. (2012) Spontaneous and Medically Induced Cerebrospinal Fluid Leakage in the Setting of Pituitary Adenomas: Review of the Literature. Neurosurgical Focus, 32, E2. https://doi.org/10.3171/2012.4.FOCUS1268

[13] Tan, J., Song, R., Huan, R., et al. (2020) Intraoperative Lumbar Drainage Can Prevent CSF Leakage during Transsphenoidal Surgery for Pituitary Adenomas: A Systematic Review and Meta-Analysis. BMC Neurology, 20, Article No. 303. https://doi.org/10.1186/s12883-020-01877-Z

[14] Patel, P.N., Stafford, A.M., Patrinely, J.R., et al. (2018) Risk Factors for Intraoperative and Postoperative CSF Leaks in Endoscopic Transsphenoidal Sellar Surgery. Otolaryngology_Head and Neck Surgery, 158, 952-960.

https://doi.org/10.1177/0194599818756272 
[15] Li, J., Zhou, K., Luo, K., et al. (2014) Relationship between Diaphragma Sellae Morphology and Residual Tumor and Complications during Nasal Endoscopic Transsphenoidal Resection of Pituitary Adenoma. Chinese Journal of Minimally Invasive Surgery, 14, 353-358.

[16] Huang, L., Guan, X., Jia, W., et al. (2014) The Treatment Strategy of a Cerebrospinal Fluid Leak during Transsphenoidal Pituitary Adenoma Surgery. Chinese Journal of Neurosurgery, 30, 1123-1125.

[17] Chen, Z., Shou, X., Shen, M., et al. (2020) Clinical Outcomes of Intraoperative Skull Base Reconstruction in Endoscopic Endonasal Skull Base Tumor Resection. Chinese Journal of Neurosurgery, 36, 2-6.

[18] Robins, J.M.W., Alavi, S.A., Tyagi, A.K., et al. (2018) The Learning Curve for Endoscopic Trans-Sphenoidal Resection of Pituitary Macroadenomas. A Single-Institution Experience, Leeds, UK. Acta Neurochirurgica, 160, 39-47. https://doi.org/10.1007/s00701-017-3355-1

[19] Ma, D., Song, W., Wang, H., et al. (2019) Strategy of Skull Base Reconstruction after Endoscopic Endonasal Approach for Skull Base Lesions with the Multi-Modality Fusion. Chinese Journal of Minimally Invasive Neurosurgery, 24, 349-352.

[20] Jiang, Z., Han, Y., Xu, D., et al. (2015) Application of Neuroendoscopy for Intraoperative and Postoperative Cerebrospinal Fluid Leaks Following Transsphenoidal Surgery for Pituitary Adenomas. Chinese Journal of Minimally Invasive Surgery, 15, 922-925.

[21] Strickland, B.A., Lucas, J., Harris, B., et al. (2018) Identification and Repair of Intraoperative CSF Leaks in Endonasal Transsphenoidal Pituitary Surgery: Surgical Experience in a Series of 1002 Patients. Journal of Neurosurgery, 129, 425-429. https://doi.org/10.3171/2017.4.JNS162451 